\title{
Myelodysplastic syndrome with myelofibrosis in a 12-year-old patient - A case report
}

\author{
Andreea Oltean ${ }^{1}$, Mihaela Ioana Chincesan ${ }^{2 *}$, Oana Marginean ${ }^{2}$, Emoke Horvath $^{3}$ \\ 1. Mures County Emergency Hospital; Pediatric Clinic I Târgu-Mureş, Romania \\ 2. University of Medicine and Pharmacy Tg-Mures; Pediatric Clinic I, Târgu Mureş, Romania \\ 3. University of Medicine and Pharmacy Târgu-Mures, Romania
}

\begin{abstract}
Myelodysplastic syndromes are a heterogeneous group of clonal disorders characterized by peripheral blood cytopenia and normal or hypercellular bone marrow with dysplasia in more than one blood cell lineage, unfavorable prognosis, and lack of response to treatment. We present the case of a 12-year-old male patient who was referred to the Hematology and Oncology Department of Pediatric Clinic I Târgu-Mures in May 2016, with splenomegaly and pancytopenia. The osteomedullary biopsy revealed myelofibrosis, discrete dysplasia of the myeloid series and megakaryocytes, blasts CD34+ approximately 10\%, which led to the diagnosis of myelodysplastic syndrome with myelofibrosis. The myeloid precursors indicated a high risk of transformation into acute myeloid leukemia, so chemotherapy associated with corticosteroids was started, leading to slight improvements. [LEm: Although myelodysplastic syndrome associated with myelofibrosis is rare at this age, despite the treatment and favorable progression in the case presented, in the absence of hematopoietic stem cell transplantation the prognosis remains unfavorable.
\end{abstract}

Keywords: myelodysplastic syndrome, pancytopenia, myelofibrosis, children

Received: $6^{\text {th }}$ July 2017; Accepted: 18 ${ }^{\text {th }}$ October 2017; Published: $22^{\text {nd }}$ November 2017

\section{Introduction}

Myelodysplastic syndromes (MDS) are a heterogeneous group of clonal disorders characterized by peripheral blood cytopenia secondary to insufficient and dysplastic hematopoiesis, with high risk to evolve into acute myeloid leukemia (AML). In contrast to adulthood, MDS are rare in childhood, constituting $4 \%$ of all hematological malignancies, with an incidence of 1.8 / one million children / year in the 0-14 year age group.
Because in contrast to adult patients, the majority of pediatric patients present more often with pancytopenia, instead of anemia only (hypocellular bone marrow), Hasle et al. proposed that diagnosis of pediatric MDS should include at least two of the following criteria: persistent unexplained cytopenia, at least morphological myelodysplasia on two-cell lines, blasts more than $5 \%$, and acquired clonal cytogenetic abnormality in hematopoietic cells. An analysis by the European Working Group of Childhood suggested that pa-

*Corresponding author: Mihaela Ioana Chincesan, University of Medicine and Pharmacy Tirgu-Mures, Romania. E-mail: mchincesan@yahoo.com 
tients with cytopenia on two or three cell lines and a blast percentage $>5 \%$ in the bone marrow have an unfavorable prognosis. $[1,2]$.

MDS progresses to acute myeloid leukemia in approximately $30 \%$ of patients [3].

Myelofibrosis is a disorder of the bone marrow in which the marrow is replaced by fibrous scar tissue. Various benign diseases and oncological disorders are related to this condition such as primary myelofibrosis, myelofibrosis secondary to thrombocythemia or polycythemia, chronic myelogenous leukemia, chronic myelomonocytic leukemia, and acute leukemia [4]. Myelofibrosis is most often related with defects in function or number of platelets and megakaryocytes [5]. MDS with myelofibrosis or hyperfibrotic MDS are very uncommon and constitute $5 \%$ of all MDS [6]. The morphological information obtained from bone marrow aspiration is complemented with histological data. The diagnostic recommendations require a biopsy to be performed in all suspected myelodysplasia in which bone marrow examination is indicated [7].

The rarity of myelodysplastic syndromes in children, especially their association with myelofibrosis, and the transformation into acute myeloid leukemia with unfavorable prognosis despite treatment, and the need to improve therapeutic guidelines is the reason why we call attention to them.

\section{Case presentation}

We describe a 12-year-old male, who had a medical history of premature birth at 33 weeks of gestation, and who first presented in 2009 at the Hematology and Oncology Department of Pediatric Clinic I Târgu-Mures, Romania, with latero-cervical lymphadenopathy and hepatosplenomegaly. Complete blood counts revealed hematocrit 30\%, white blood count (WBC) 4000/ $\mu 1$ and platelets $157000 / \mu 1$. The computed tomography examination revealed mediastinal and late- ro-cervical lymph nodes, also hepatosplenomegaly, and subsequently he was transferred to the Pediatric Surgery for a lymph node biopsy. The histopathological examination revealed lymph nodes with reactive changes. Subsequently, the patient did not present for further investigations.

In May 2016 the mother addressed initially with the boy to the territorial Emergency Department complaining of vomiting, nausea, loss of appetite, marked fatigue and headache, where he was diagnosed with marked splenomegaly and pancytopenia, and he was referred to the Hematology and Oncology Department of Pediatric Clinic I Târgu-Mures for further investigations. The clinical examination on presentation revealed a poor state of nutrition with hypotrophy in weight and stature, paleness, visible collateral circulation on the arms, legs, thorax and abdomen, an ecchymosis on the left knee, fat tissue poorly represented on the thorax, arms and legs, liver palpable at $3 \mathrm{~cm}$ below the costal margin, and the spleen palpable at $12 \mathrm{~cm}$ below the left costal margin.

Laboratory findings on admission revealed a mild hypoplasia of the blood cell lines (Leucocytes $=3900 / \mu 1$, Granulocytes $=2900 / \mu 1$, Erythrocytes $=3390000 / \mu 1$, Hemoglobin $=6.8 \mathrm{~g} / \mathrm{dl}$, Hematocrit $=28.6 \%$, Mean Corpuscular Volume $(\mathrm{MCV})=73.3 \mathrm{fL}$, Platelets $=101000 / \mu 1$, Reticulocytes of 24\%o), and a blood smear with marked hypochromia, anisopoikilocytosis, microcytes, ovalocytes, schisocytes, dacryocytes, macrotrombocytes. A bone marrow (BM) examination was performed, the aspirate showing average/ normal cellularity, granulocytic series with all stages of maturation, erythroblastic series with signs of iron deficiency, and rare megakaryocytes. The patient subsequently developed pancytopenia (lowest values of Platelets $=72000$ / $\mu 1$, Leucocites $=2600 / \mu 1$, Granulocytes $=2030 /$ $\mu 1$, Hemoglobin $=6.8 \mathrm{~g} / \mathrm{dl}$ ).

As during the progression of the disease the patient presented pancytopenia, corroborated 
with marked splenomegaly, and the aspect of medullary aspiration, we decided to perform a bone marrow biopsy (BMB). The microscopic and histological description of the BMB revealed slightly higher cellularity (Figure 1) due to a proliferation of the granulocytic series that shows all the stages of maturation, with myeloid precursors $\mathrm{CD} 34^{+}$with abnormally localized immature precursors (ALIP) (Figure 2). Erythroid islands were normally represented. The characteristic location of megakaryocytes was distinguished with CD61 reaction, showing signs of dysplasia (Figure 3). Blasts/equivalents of $\mathrm{CD}_{34}{ }^{+}$blasts were approximately $10 \%$. Rich vascular stroma and proliferation of reticulin fi- bers were present in the form of an irregular network with unevenly thickened fibers (Grade 2) (Figure 4), the conclusion being: myelodysplastic syndrome - Refractory Anemia with Excess Blasts (RAEB) associated with myelofibrosis.

During hospitalization, imaging investigations were conducted as well. They revealed a marked splenomegaly, discrete hepatomegaly and pancytopenia (Vitamin B12 and folic acid levels in serum were within the normal range). The computed tomography of the brain showed no brain lesions, the abdomen and pelvis tomography revealed hepatomegaly, splenomegaly $(180 / 78 \mathrm{~mm})$, a dilation of the spleen vein of 12 mm, multiple millimeter lymph nodes (celiac,

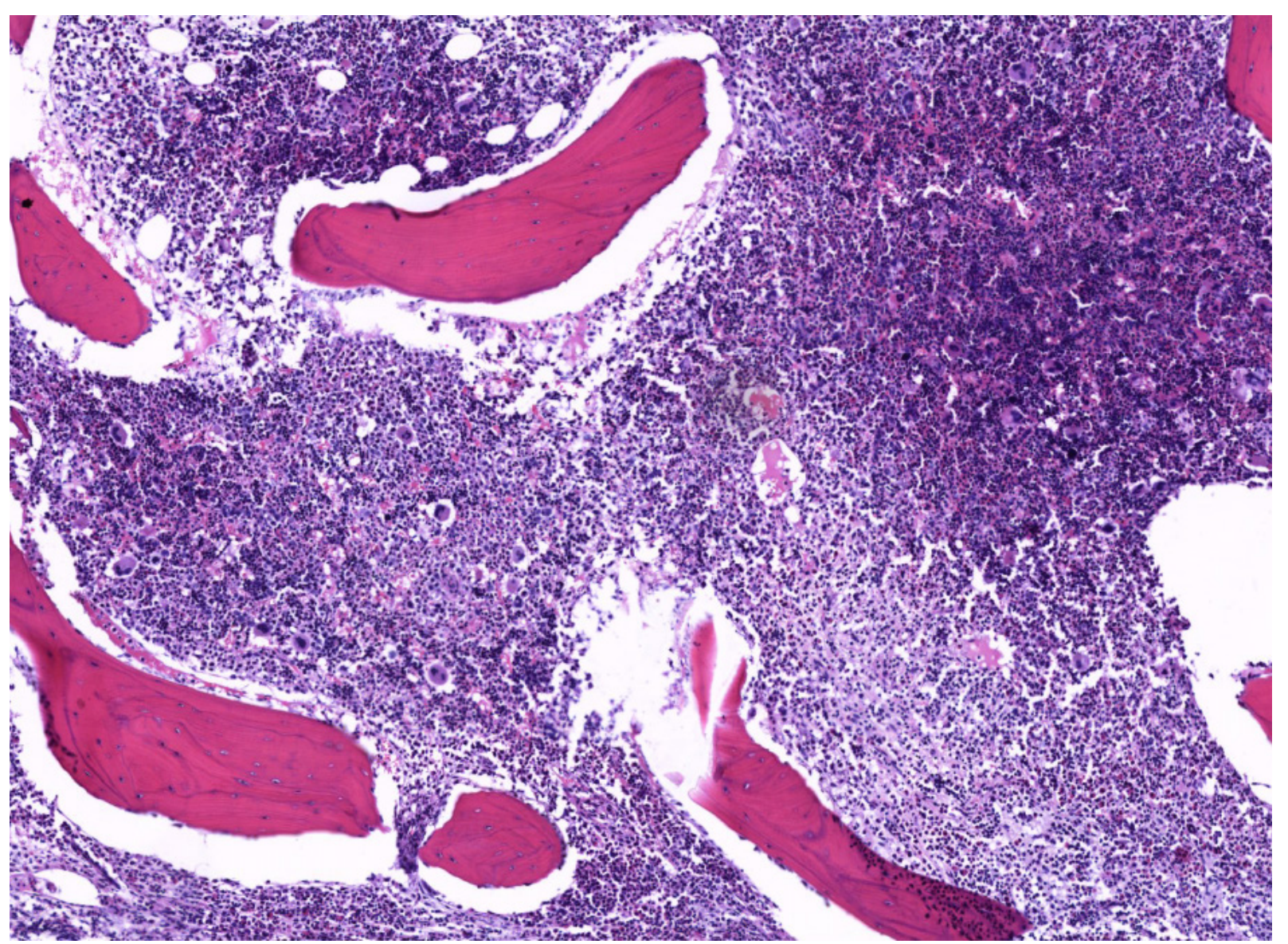

Fig. 1. Bone marrow biopsy: hypercellular marrow with granulocytic proliferation, Hematoxylin Eosin (H\&E) stain, $5 \times$ magnification 


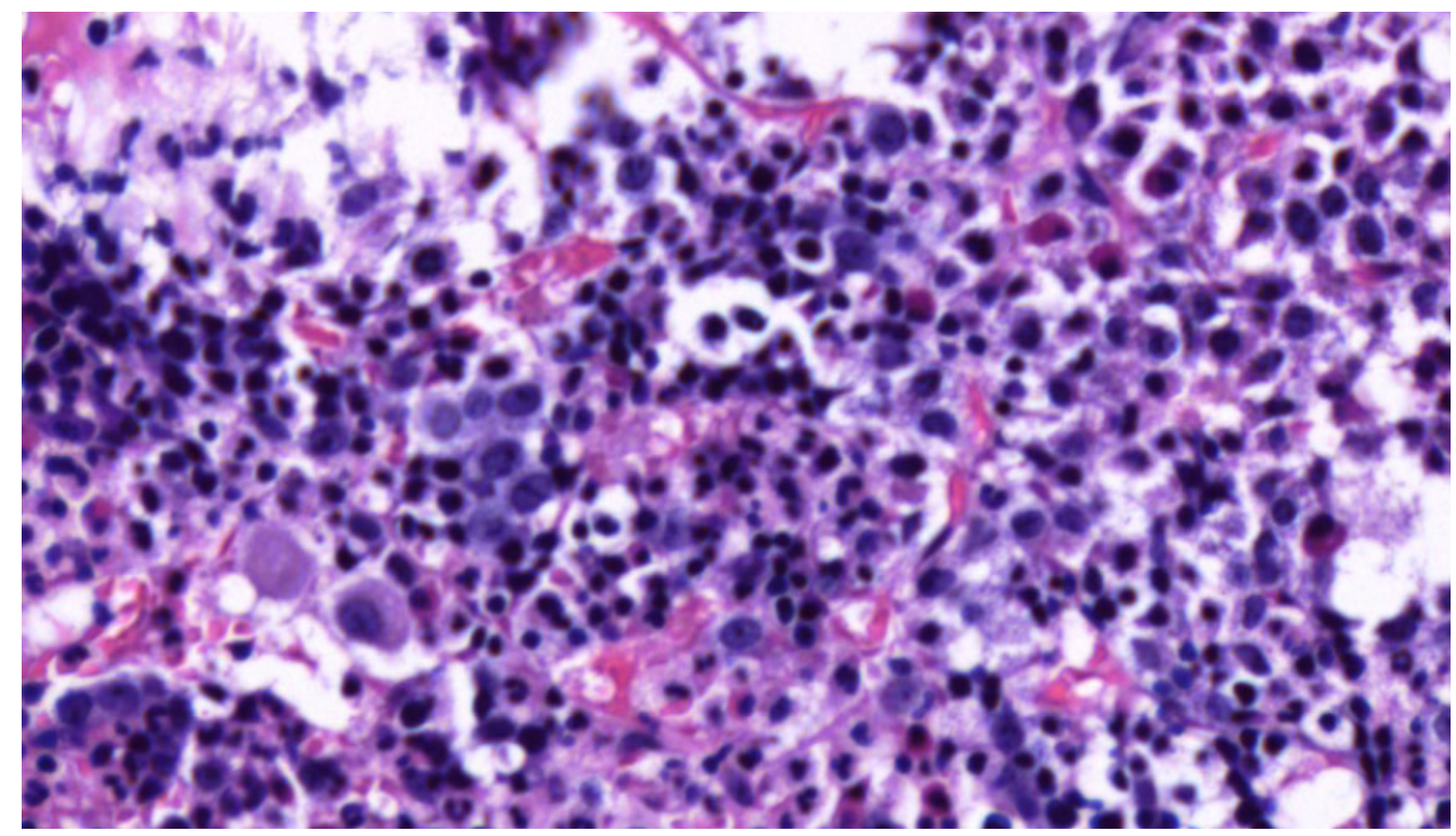

Fig. 2. Bone marrow section with abnormal localization of immature precursors, H\&E stain, $40 \times$ magnification

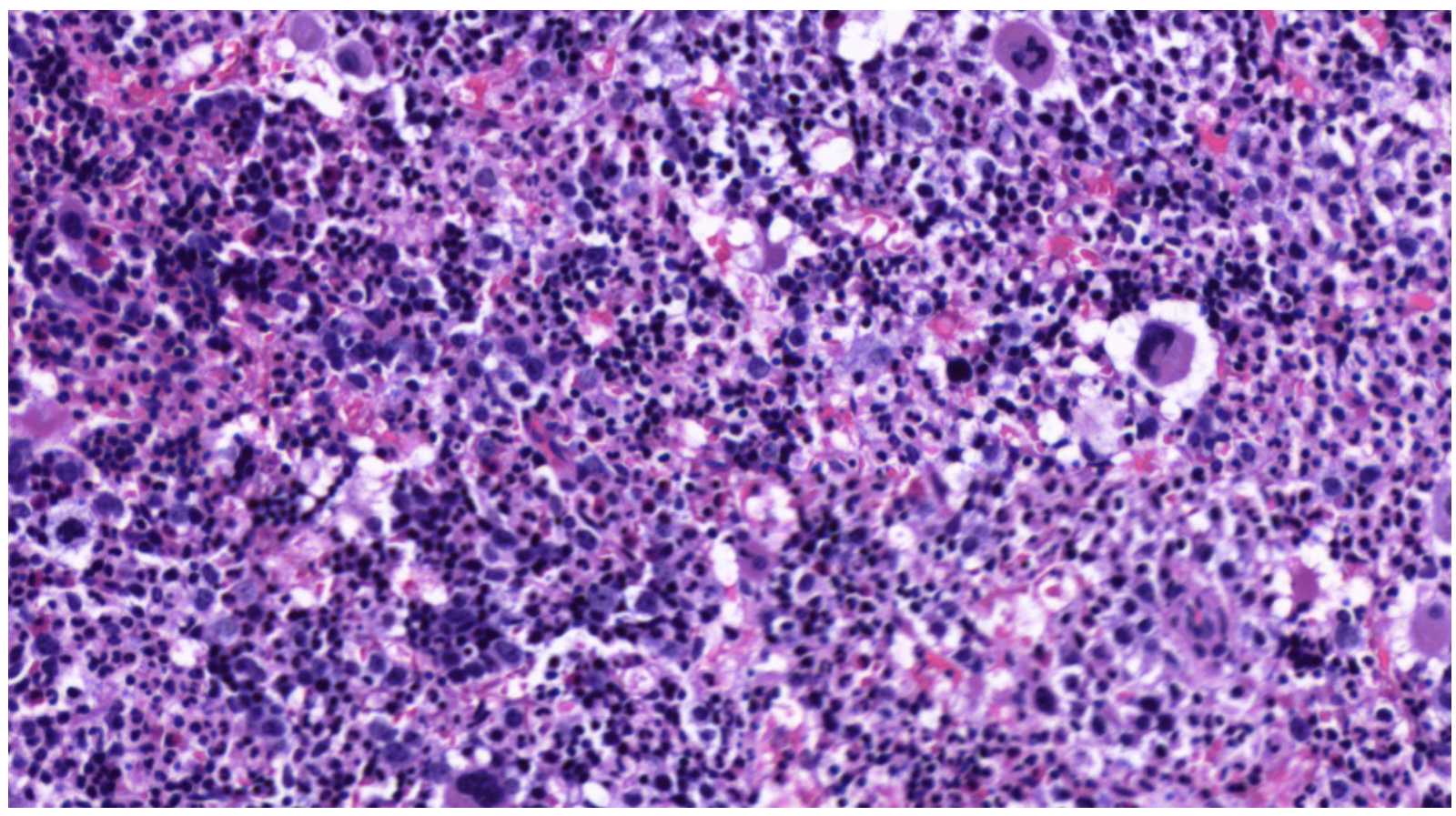

Fig. 3. Bone marrow tissue with megakaryocytes of various size and hypolobulated nuclei.

H\&E stain, $20 \times$ magnification 


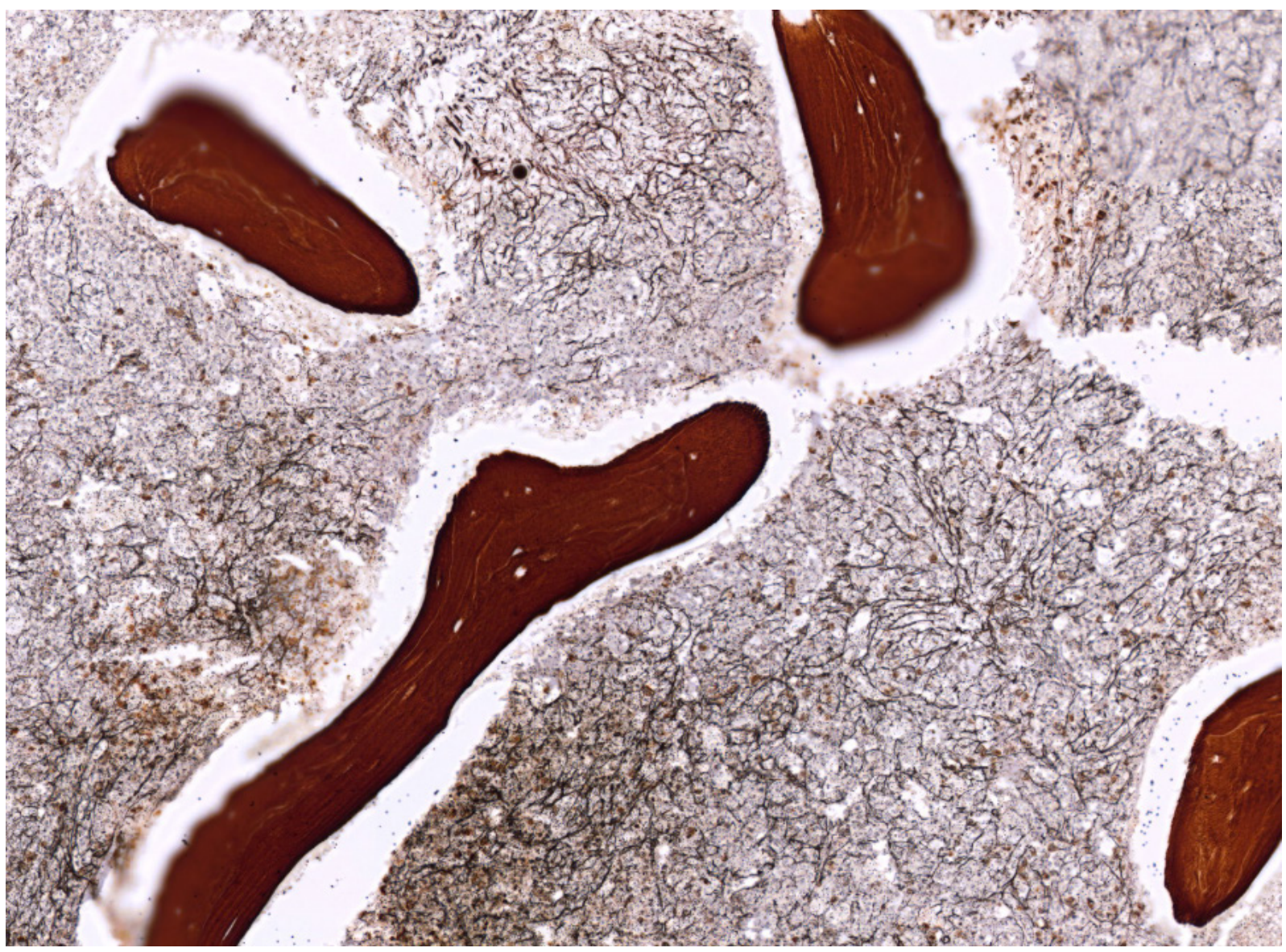

Fig.4. The micrograph shows a grade 2 fibrosis: reticulin fibres are diffusely increased with extensive intersections (Gömöri silver stain, 10 x magnification)

retroperitoneal), and the demineralization of the pelvic bones.

For the differential diagnosis of splenomegaly we excluded the following conditions: thalassemia based on Hemoglobin (Hgb) electrophoresis (Hgb A 97.6 \%, Hgb A2 $2.4 \%$, Hgb F 0.0\%), storage diseases - Gaucher and Niemann-Pick disease (beta-Glucosidase, acid sphingomyelinase and beta-galactosidase within the normal range), inflammatory syndrome and colagenosis (Anti-double-stranded DNA antibodies, Anticardiolipin IgG antibodies, Anti-nuclear antibodies, Lupus Cells, Circulating Immune Complexes, Antistreptolysin O Rheumatoid Factor - all with- in the normal range), infectious diseases (negative blood cultures, pharyngeal exudate - negative, TORCH serology - negative). Differential diagnosis was also performed for lymphoproliferative disorders based on bone marrow aspiration, and for myeloproliferative disorders (neuroblastoma: Vanillylmandelic Acid [VMA] was determined from urine per 24 hours VMA=2.19 $\mathrm{mg} / 24 \mathrm{~h}$ [normal value $<6.60$ ] and Neuron Specific Enolase [NSE] $9.21 \mathrm{ng} / \mathrm{mL}$ [normal value $<16.30]$ ).

The cytogenetic bone marrow examination revealed a normal 46, XY karyotype. Molecular biology tests were also performed, the studied 
mutations being: FLT3 (FMS-like tyrosine kinase 3), ITD (Internal Tandem Duplication), and D835 (of codon 835); NPM1 (nucleophosmin 1) mutation type A was positive.

Based on the fact that the patient had pancytopenia, myelofibrosis, dysplasia of the myeloid series and megakaryocytes and blasts/equivalents of $\mathrm{CD}^{2} 4^{+}$blasts approximately $10 \%$, the final diagnosis was myelodysplastic syndrome with myelofibrosis. The presence of myeloid precursors $\mathrm{CD}_{34}{ }^{+}$with atypical localization being associated with high risk of transformation into acute myeloid leukemia, chemotherapy was initiated with low doses of intravenous ARA-C (cytosine arabinoside) associated with corticosteroids. Following the treatment, the patient's overall condition improved, gaining about $5 \mathrm{~kg}$, with less visible collateral circulation, the dimensions of the spleen reducing for a period of time with each cycle of chemotherapy.

On reevaluation ( 8 months later, in March 2017) after 6 cycles of chemotherapy associated with corticosteroids, the BMB indicated osteomedullar tissue with increased cellularity, with regenerative changes of the myeloid series. Megakaryocytes were present with signs of dysplasia. Myeloid series with the presence of all maturation steps, regression of the marrow fibrosis CD34, and CD117 immunostains revealed less than $5 \%$ blasts. These results suggest a favorable outcome, which might be transient. Considering that MDS with fibrosis have an unfavorable prognosis, despite the remission under chemotherapy there is a risk of relapse and transformation into acute myeloid leukemia, so curative treatment would require allogenic bone marrow transplantation.

\section{Discussion}

On average, $30 \%$ of patients with MDS present increased risk of AML transformation. One of the important morphological features related to disease progression in MDS is the presence of significant fibrosis (grade 2 or 3 ), reported as an independent prognostic factor. A high BM cellularity, multilineage dysplasia associated with abnormally localized immature precursors (ALIPs) have been often seen in leukemic transformation [8]. These morphological features were present in our case.

MDS and other myeloproliferative disorders are rare entities, particularly in children. The transition between the forms of myelodysplastic syndromes is very fluid, having the following features: anemia, bicytopenia, pancytopenia without any known cause, dyserythropoiesis with anisocytosis, dysgranulopoiesis with hyposegmentation and hypogranulation of granulocytes, and dysmegakariopoiesis with micromegakaryocytes [9].

MDS with myelofibrosis represents a distinct clinicopathological entity, and marrow fibrosis seems to confer a poor prognosis [6].

In our case, the patient had normal blood count on the first presentation, but 7 years later he had both anemia and thrombocytopenia. The big time gap is because he had not presented for further investigations. It is well known that after years of BM insufficiency with a more or less pronounced deficit in all three cell series, patients pass into a phase of insidiously increasing blast counts [9]. Morphological evaluations of peripheral blood and of bone marrow are important components in the evaluation of pediatric myelodysplasia [10]. In our case, bone marrow showed no signs of marrow failure, but the BMB indicated myelofibrosis, with dysplasia, and a blast count of $10 \%$.

In an English study on 887 pediatric patients with myelodysplastic syndrome from 13 published series, $68.7 \%$ were idiopathic/de novo, $23.9 \%$ were associated with constitutional/inherited disorder, and $7.4 \%$ were therapy related [11]. Cytostatic treatment for neoplasia may play a role in the development of secondary myelodysplastic syndrome [12]. 
In our case, the etiology of the myelodysplastic syndrome remains unknown as well.

At the time of his first and second presentation, the patient had splenomegaly caused by extramedullary hematopoiesis. When confronting with splenomegaly, the differential diagnosis must include: spleen malformations, hematoma or rupture, thalassemia major, hereditary spherocytosis, Glucose-6-Phosphate Dehydrogenase (G6PD) deficiency, severe hemolytic anemia, myeloproliferative diseases, chronic infections, viral infections (cytomegalovirus, hepatitis A, B, Epstein-Barr virus), colagenosis, leukemia, lymphoma, storage diseases [13]. All these causes were taken into consideration.

Bone marrow failure manifested by pancytopenia is an important indication for bone marrow examination. The etiologies of cytopenia are different; in pediatric patients, inherited bone marrow failures being major causes of bone marrow failure [14]. In our case, the bone marrow aspirate was normal in contrast with the pancytopenia that led us to the BMB that revealed myelofibrosis associated with MDS. This disease progresses slowly, and the typical clinical features include massive splenomegaly, progressive anemia and systemic symptoms. It was found that in myelodysplastic syndrome, the occurrence of ALIP, but also the presence of $\mathrm{CD} 4^{+}$cell aggregates, is associated with an increased risk of leukemic transformation [15]. In our case, the BMB performed showed not only myelofibrosis but the presence of myeloid precursors as well as CD $34^{+}$which imposed performing cytogenetic and molecular examination. Cytogenetic results have an important role in the diagnosis and identification of prognostic subgroups of MDS [16].

An increasing number of acquired clonal cytogenic abnormalities with prognostic significance have been described in hematopoietic cells. In a British study on 124 patients with myelodysplastic syndrome, karyotype abnormalities were found in $36 \%$ of the patients; reduced sur- vival was associated with the presence of either single or multiple clonal abnormalities of chromosome 7 or 8 [17]. A comparative study based on the accumulation of chromosomal changes in MDS demonstrated that $77 \%$ of MDS cases with cytogenetic evolution progress to AML in comparison to $30 \%$ of patients without cytogenetic evolution [18].

The cytogenetic examination performed in the presented case did not detect any numerical or structural chromosome abnormalities, but it requires repetition if the disease progresses. Molecular biology tests have revealed the NPM1 mutation; this mutation has a $50 \%$ frequency in myeloid acute leukemia, and in adults, it is associated with a good prognosis in the absence of mutation FLT3 - ITD.

In pediatric patients, the NPMI mutations merits further studies, in order to determine their individual contribution to the pathogenesis of childhood MDS/AML, and their comprehensible relation to prognosis.

Secondary to cytopenias, patients with MDS are at increased risk of bleeding, infections, and transfusion-dependent anemia. Even if there is an uncertain risk of transformation to AML, deaths are secondary to bone marrow failure. The treatment in MDS is most often symptomatic, and focuses on preventing or reducing complications of the disease, because there is no truly effective treatment for this condition [19]. In MDS with myelofibrosis, chemotherapy has a reduced efficacy, but allogeneic HSCT (hematopoietic stem cell transplantation) or other new agents may be effective, but an additional analysis is required [20].

\section{Conclusions}

MDS is a rare condition in childhood that often progresses to AML. Facing with a patient with both pancytopenia and splenomegaly, the differential diagnosis must always exclude my- 
elodysplastic syndrome. Bone marrow aspirate is not always enough to clarify the etiology of a pancytopenia, the bone marrow biopsy being able to give more details about bone marrow failure. However, in most cases, it is impossible to make a difference between reactive, drug related atypias in hematopoietic cells (reactive changes) and residual myelodysplasia in a bone marrow biopsy. Due to the fact that MDS with myelofibrosis are very uncommon in children, and marrow fibrosis confers a poor prognosis which markedly shortens the survival time in the clinical evolution of the disease - suggesting that conventional treatment is no longer effective unless HSCT is performed, we consider that HSCT is highly indicated in this case.

\section{Abreviations}

ALIP = Abnormally Localized Immature Precursors

$\mathrm{AML}=$ Acute Myeloid Leukemia

ARA-C $=$ Cytosine Arabinoside

$\mathrm{BM}=$ Bone Marrow

$\mathrm{BMB}=$ Bone Marrow Biopsy

$\mathrm{CML}=$ Chronic Myelogenous Leukemia

G6PD = Glucose-6-Phosphate Dehydrogenase

$\mathrm{Hgb}=$ Hemoglobin

HSTC $=$ Hematopoietic Stem Cell Transplantation

$\mathrm{MCV}=$ Mean Corpuscular Volume

MDS = Myelodysplastic Syndrome

$\mathrm{NSE}=$ Neuron Specific Enolase

$\mathrm{VMA}=$ Vanillylmandelic Acid

$\mathrm{WBC}=$ White Blood Count

\section{Conflict of interests}

The authors declared the absence of conflict of interests.

\section{References}

1. Elghetany TM, Bhatla T. Myelodysplastic Syndromes and Myeloproliferative Disorders. Lanzkowsky P. Hofmann I. Jeffrey M. Lipton Lanzkowsky's Manual of Pediatric Hematology and Oncology. Sixth Edition Elsevier. 2016, London, 348-351.

2. Hasle H, Niemeyer CM, Chessells JM. A pediatric approach to the WHO classification of myelodysplastic and myeloproliferative diseases. Leukemia. 2003;17(2):277-282. DOI: 10.1038/sj.leu.2402765

3. Disperati P, Ichim CV, Tkachuk D. Progression of myelodysplasia to acute lymphoblastic leukaemia: implications for disease biology. Leuk Res. 2006;30:233239. DOI: 10.1016/j.leukres.2005.06.011

4. Vardiman JW, Thiele J, Arber DA, Brunning RD, Borowitz MJ, Porwit A, et al. The 2008 revision of the World Health Organization (WHO) classification of myeloid neoplasms and acute leukemia: rationale and important changes. Blood. 2009;1145(5):937-51. DOI: 10.1182/blood-2009-03-209262

5. Tefferi A. Pathogenesis of myelofibrosis with myeloid metaplasia. J Clin Oncol. 2005;23(33):8520-30. DOI: 10.1200/JCO.2004.00.9316

6. Das P, Deepali J, Das R, Garewal G. Hyperfibrotic myelodysplastic syndrome: a report of three cases from north India. Turk J Hematol. 2009;26:93-6.

7. Bowen D, Culligan D, Jowitt S, Kelsey S, Mufti G, Oscier D, et al. Guidelines for the diagnosis and therapy of adult myelodysplastic syndromes. Br J Haematol. 2003;120(2):187-200. DOI: 10.1046/j.13652141.2003.03907.x

8. Porwit A, Saft L. The AML-MDS interface-leukemic transformation in myelodysplastic syndromes. J Hematopathol. 2011;4:69-79. DOI: 10.1007/s12308-0110088-6

9. Diem H. Myelodysplasia. Tehml H, Diem H, Haferlach T. Color Atlas of Hematology - Practical Microscopic and Clinical Diagnosis. 2nd revised edition 2004. Munich, 106-9.

10. Glaubach T, Robinson L.J, Corey S.J Pediatric Myelodysplastic Syndromes: They Do Exist! J Pediatr Hematol Oncol. 2014;36:1-7. DOI: 10.1097/ MPH.0000000000000046

11. Elghetany TM. Myelodysplastic syndromes in children: a critical review of issues in the diagnosis and classification of 887 cases from 13 published series. Arch Pathol Lab Med. 2007;131:1110-6.

12. Cîrstea M, Coliță A, Ionescu B, Ghiaur A, Vasilescu D, et al. Therapy-related myelodysplastic syndrome after 
successful treatment of acute promyelocytic leukemia: case report and literature review. Rev Romana Med Lab. 2017;25(2):165-79. DOI: 10.1515/rrlm-20170013

13. Camitta BM. Diseases of blood: Splenomegaly. Kliegman RM. Behrman RE, Jenson HB, Waldo E, Nelson Textbook of Pediatrics. 18th edition, Philadelphia, Elsevier, 2008, 2091-2.

14. Leguit RJ, Van Den Tweel J.G. The pathology of bone marrow failure. Histopathology. 2010;57(5):655670. DOI: 10.1111/j.1365-2559.2010.03612.x

15. Bănescu C, Benedek I, Duicu C, Demian S, Voidăzan $\mathrm{S}$. Cytogenetic findings and their prognostic impact in myelodysplastic syndrome patients. Rev Romana Med Lab. 2011;19(2):139-47.

16. Della Porta MG, Malcovati L. Myelodysplastic syndromes with bone marrow fibrosis, Haematol Hematol
J. 2011;96:180-3. DOI: 10.3324/haematol.2010.039875

17. Geddes AD, Bowen DT, Jacobs A. Clonal karyotype abnormalities and clinical progress in the myelodysplastic syndrome, Br J Haematol. 1990;76(2):194202. DOI: 10.1111/j.1365-2141.1990.tb07871.x

18. Bernasconi P, Klersy C, Boni M. Does cytogenetic evolution have may any prognostic relevance in myelodysplastic syndromes? Ann Hematol. 2010;89:545551. DOI: $10.1007 / \mathrm{s} 00277-010-0927-\mathrm{Z}$

19. Koppel A, Schiller G. Myelodysplastic syndrome: an update on diagnosis and therapy. Curr Oncol Rep. 2008 10(5):372-378. DOI: 10.1007/s11912-008-0058-z

20. Xiaojun KS, Xu Min L. Hypocellular myelodysplastic syndrome with myelofibrosis in acute myeloid leukemia transformation: A case report. Oncol Lett. 2015;10:422-4. 